\title{
Stability Cogitated Electric Vehicle Charging Infrastructure Planning
}

\author{
C.H. Dharmakeerthi, Student Member, IEEE, and N. Mithulananthan, Senior Member, IEEE
}

\begin{abstract}
Electrification of the transportation is taking place at an accelerated rate. Even though, electric vehicles (EV) evidently bring numerous environmental and economic benefits, their impact on power systems should not be overlooked. It has been identified that EV load characteristics can significantly affect power system voltage stability and small signal stability. Hence, it is important to consider mitigating of stability impacts right from the planning stage of bulk EV charging stations. However, unavailability of suitable stability evaluating indexes that could fit into planning algorithms is a hindrance. This study proposes two computationally efficient indexes to compare stability status in different planning options. The developed indexes have been tested, verified and utilized in a multi objective planning algorithm to identify a comprehensive solution, which satisfies the grid operator, EV customer and the charging facility investor optimally.
\end{abstract}

Index Terms-- Eigenvalue analysis, electric vehicle, EV charging stations, loading margin, oscillatory stability, power system planning, steady state voltage stability.

\section{INTRODUCTION}

$\mathrm{D}$ EPLETING fossil fuel resources, environmental and health problems associated with vehicular emissions and the energy security concerns have highlighted the requirement of electrified transportation. Hence, the countries around the globe provide EV consumers with abundant financial and non-financial incentives to make EV popular [1, 2]. These efforts have resulted in a rapid electrification of the transportation as reflected in recent increment in global EV sales. According to [1] the global EV sales have increased by twofold during 2011 and 2012. One unavoidable concern associated with this transformation is the grid impacts of EV charging.

Researchers have identified several grid impacts associated with EV grid integration. Overloading of primary system components, voltage regulatory limit violations, increased system losses and harmonic issues are among them [3]. Further, EV charging impacting power system oscillatory stability and voltage stability have also been reported in [4-10].

Manuscript received March 7, 2017; revised March 29, and April 9, 2017; accepted April 19, 2017

C. H. Dharmakeerthi is with the School of Information Technology and Electrical Engineering, University of Queensland, Australia and Ceylon Electricity Board, Sri Lanka (e-mail: champa80@yahoo.com).

N. Mithulananthan is with the School of Information Technology and Electrical Engineering, University of Queensland, Australia (e-mail: mithulan@itee.uq.edu.au)
Therefore it is wise to consider the issues during charging station planning so that potentially expensive remedies can be completely avoided or delayed.

A number of planning strategies have been introduced with different objectives in recent literatures for a successful transformation to an electrified transportation system. The objectives of the planning study given in [11] are to minimize charging station construction and operation costs and the accessing cost to the charging facility. The planning study in [12] identifies the location of the charging station based on the locations of residential communities, refuelling stations, parking lots and power transmission stations. The strategy given in [13] aims providing services to most customers from the given investment, while [14] intends to serve the customers reliably. Further, the studies in [15-18] consider minimization of investment and operational cost of EV charging, while grid limitations have been considered within the planning constraints. The planning study in [19] has concentrated on maximum utilization of the charging station with lower power losses and lower voltage deviations. It is evident that most of the planning objectives are traditionally aiming to satisfy the requirements of EV consumers or investors [11] [12], [15], [2024]. Only scant attention has been given to the power grid aspects in planning studies so far. However, a comprehensive planning strategy should consider not only geographical and economical aspects, but also the technical aspects of the power grid.

Stability cogitated system planning become important as system instabilities become more frequent, with the maximum utilization of the system assets in modern power systems. However, system stability has not been considered in most of the present planning studies. Unavailability of suitable stability evaluating indexes to incorporate within planning algorithms suggests the importance of deriving computationally efficient indexes which are having good physical interpretations. Hence, this study introduces two indexes to identify oscillatory stability and voltage stability preserves planning solution. The indexes are validated analytically and numerically. Finally, the derived indexes are utilized in an EV charging infrastructure planning task to build charging facilities which not only satisfies the requirements of EV customer and the charging facility investor, which should also preserve a secured and economical grid operation.

The rest of the paper is organized as follow. Section II describes EV load model and its influence on power system stability. The theoretical background relating oscillatory and static voltage stabilities is introduced in Section III. Section IV develops and validates two indexes to examine stability impact of EV loads, while section V reports a planning case study and 
the results. Finally, Section VI provides conclusions of the paper.

\section{EV LOAD MODEL}

The EV load is found to be having characteristics which combines negative exponential and constant power load behavior, as shown in (1) [7] .

$$
\frac{P}{P_{o}}=a\left(\frac{V}{V_{o}}\right)^{-\alpha}+b
$$

The negative exponential component has been resulted from the resistances present in the ac to dc converter, ac filter and the lead conductor. Studies show that the higher the resistances the bigger the negative exponential load component [7]. A load model which is based on $1 \mathrm{~m} \Omega$ filter inductor parasitic resistance, $2 \mathrm{~m} \Omega$ switch turn on state resistance and $0.02 \Omega$ lead conductor resistance [7] is incorporated in this study. The load model parameters are $a=0.07, b=0.93$ and $\alpha=3.1$, as per (1).

The constant power load increase its current consumption at lower system voltages while the negative exponential load component increase its power consumptions at lower system voltages. Hence, the both load components contribute to degrade system voltage stability significantly during a voltage instability incident.

The EV load is found to be affecting the system voltage stability negatively as reported in $[4,6,7]$. On the other hand, studies in $[4,5,10]$ have identified that $\mathrm{EV}$ charging affects system oscillatory stability. Therefore, from power utility point of view, it is important to consider these issues during charging station planning.

\section{THEORETICAL BACKGROUND}

Basic theory behind the oscillatory and static voltage stability is explained in this section before introducing the new indices developed for stability cogitated planning.

\section{A. Oscillatory Stability}

The power system oscillatory stability describes the ability of the system to remain synchronism when undergoes small disturbances [25]. Understanding of the problem requires studying of the electromechanical oscillations in the system. Generation and load changes, fast exciters, negative interaction among controllers are contributing to system oscillations [26]. The factors that determine the system response to such system variations include the initial operating point, type of excitation control and the strength of the transmission system [25].

The oscillations should be damped, as sustained oscillations cause excessive wear and fatigue in power system components. Furthermore, these oscillations could grow in amplitude and if not controlled they could lead to instability and blackouts. The Eigenvalue analysis methodology for identifying system oscillatory stability status is described below.

The power system dynamics can be described by a set of first order differential equations and a set of algebraic equations as shown below.

$$
\begin{aligned}
& \dot{x}_{i}=f_{i}(x, y, u) \\
& 0=g_{i}(x, y)
\end{aligned}
$$

The $x_{i}, y_{i}$ and $u_{i}$ are system state variables, algebraic variables and input variables, respectively, while $f_{i}$ and $g_{i}$ are non-linear functions. If the system equilibrium state is disturbed by a small perturbation, the first order Taylor series expansion can be applied to obtain a linear approximation to above expressions by neglecting the higher order terms, as shown below.

$$
\begin{aligned}
\Delta \dot{x} & =A \Delta x+B \Delta y+E \Delta u \\
0 & =C \Delta x+D \Delta y
\end{aligned}
$$

The $A$ is known as the system state or the plant matrix. Eliminating $\Delta y$ from (4), will result

$$
\Delta \dot{x}=A_{s y s} \Delta x+E \Delta u
$$

where,

$$
A_{s y s}=A-B C D^{-1}
$$

Eigenvalue analysis of $A_{s y s}$ is carried out to determine the stability of the system. Eigenvalues $\left(\lambda_{i}\right)$ can either be real or complex. Real Eigenvalues represent non-oscillatory modes. Positive real Eigenvalues represents aperiodic instability. Negative real ones represent decaying modes. The larger the magnitude is the faster the decay.

Complex Eigenvalues represent oscillatory modes. They are appearing as conjugate pairs. The associated damping ratios $\zeta i$ of them can be described by,

$$
\begin{aligned}
& \lambda_{i}=\sigma_{i}+j \mu_{i} \\
& \zeta_{i}=\frac{-\sigma_{i}}{\sqrt{\left(\sigma_{i}^{2}+\mu_{i}^{2}\right)}}
\end{aligned}
$$

The negative $\boldsymbol{\sigma}_{i}$ values represent damped oscillations, while the positive values corresponding to oscillations growing in amplitude. The smaller the damping ratios $\left(\zeta_{i}\right)$ the lower the system stability. Modes of oscillation can be identified by analyzing participation factors.

\section{B. Voltage Stability}

Voltage stability of a power system is preserved if acceptable voltages are maintained in all network busses during normal operating conditions and after being subjected to a disturbance [25]. During a voltage instability event the system voltages decline progressively and uncontrollably. This happens majorly when system generation and transmission are unable to meet the system reactive power demand. Heavily loaded lines, system contingencies, increased reactive power consumption of the loads, limitations in reactive power generations and actions of voltage control devices are some of the main factors which affect voltage instabilities. Performance indexes are important to determine proximity to voltage collapse. A widely accepted voltage stability assessment index, the static loading margin is described below.

A quasi-steady state description of a power system which is applicable to voltage stability analysis can be described by a set of differential and algebraic equations as given in (9),

$$
\begin{aligned}
& \dot{x}=f(x, y, \lambda) \\
& 0=g(x, y, \lambda)
\end{aligned}
$$


The state variables are represented by $x$, while y represents the algebraic variables. The $\lambda$ represent a parameter or set of parameters which are slowly changing with time. As the $\lambda$ changes system moves from one equilibrium point to another until it reaches the collapse point. The set of equations in (9) can also be represented by (10), alternatively.

$$
\left[\begin{array}{l}
\dot{x} \\
0
\end{array}\right]=F(x, y, \lambda)
$$

An equilibrium point $\left(x_{0}, y_{0}, \lambda_{0}\right)$ can be characterized by,

$$
F\left(x_{0}, y_{0}, \lambda_{0}\right)=0
$$

Based on the non-singularity assumption of algebraic equations, an equilibrium point where the Jacobian of (9) is singular, is known as a singular bifurcation point $\left(x^{*}, y^{*}, \lambda^{*}\right)$ [27]. If the system load is taken as the parameter which varies slowly, loading margin or the static voltage stability margin $(S V M)$ can be achieved by (12). The loading parameter at the current operating point is denoted by $\lambda_{0}$.

$$
S V M=\lambda^{*}-\lambda_{0}
$$

The loading margin is the additional load increment (increased in a specific pattern) required to drive the system towards instability from the current operating point. The pattern how the system loads are increased and how the system generators are arranged to respond to that load increment is defined by the load and the generation directions, respectively. Loading margin identifies how close the current operating point to the voltage collapse point. It is an accurate index that considers the system nonlinearities and limits like the generator reactive power limits, the line MVA flow limits etc, which are encountered during system load increments.

The continuation of power flow (CPF) method is widely incorporated to determine the static loading margin. It incorporates an iterative method consists of a predictor and corrector to generate the PV curve. The nose of the curve represents the voltage collapse point. A static voltage stability study is carried out in this research using PSAT (Power System Analysis Toolbox). Loading margin has been utilized to compare the performance of the proposed index (QRPI).

\section{DeVelopment of Stability InDEXeS}

The indexes for oscillatory stability and voltage stability cogitated planning are described in this section. The performances of the indexes have been verified through established stability evaluating techniques.

\section{A. Index for oscillatory stability}

It is important to evaluate impact of EV load on oscillatory stability during charging station planning, as it is a bulk system load. The Eigen value analysis and the time domain simulations are the most widely used oscillatory stability analysis methodologies. These methodologies require detailed dynamic representation of system components. However, most system planning studies are based on steady state system analysis. Therefore, an index which can be incorporated in static planning studies to identify a better oscillatory stable placement has been evaluated in this research. The system oscillatory behavior is affected by the equilibrium point, which can be studied with steady state analysis. Hence, the significance of operating point in determining system oscillatory stability is taken into consideration in deriving the index.

System oscillatory stability is determined by many factors and initial operating point is dominant among them. It is evident that when power systems are stressed or operating closer to their limits they are vulnerable to become unstable even due to a smaller system disturbance. Hence, it is advantageous to identify a quantity that can relate the influence of steady state operating point to the system oscillatory stability status. Any system planning strategy which relies only on steady state system analysis can utilize that quantity to identify oscillatory stability status of different planning solution. This research has introduced an index by considering the relationship between the network bus angles and the damping ratio of the critical modes. The index is based on the sum of the individual bus voltage angle deviations of the system buses with respect to the system reference bus angle.

There are few studies which link bus voltage angle deviation to system stability. The study in [28] incorporates bus voltage angle information to develop a methodology to build a two bus equivalent of the whole network using synchrophasor data, to determine distance to the steady state stability limits. The study in [29] follows the same concept to describe how the angle information from PMU data can be utilized to monitor system voltage stability status. An index called integral square generator angle index has been introduced in [30] for contingency ranking and screening. Adapting the same method [31] proposes integral square bus angle index for predicting instability and for designing remedies to loss of synchronism. The index takes the time integral of the summation of the weighted and squared angle deviations in selected network buses.

The new index introduced in this study is named as oscillatory stable placement index ( $O S P I)$, which can be utilize to evaluate relative voltage stability status in different planning solution. The voltage angle of a system bus $\left(\theta_{b u s, i}\right)$ is a relative quantity. The total of individual bus angle deviations of the system busses with respect to the slack or the infinite bus angle $\left(\theta_{i n f}\right)$, described in (13), is a fixed value for a steady system operation point.

$$
\text { OSPI }=\sum_{i=1}^{l}\left(\theta_{\text {bus }, i}-\theta_{\text {inf }}\right)
$$

A higher positive $O S P I$ indicates a better oscillatory stable case, on the other hand higher negative indicates a worse case. The validity of $O S P I$ to determine relative oscillatory stability status has been proved analytically and numerically by verifying relationship between the $O S P I$ and the damping ratio of the critical modes in the following section.

\section{1) Analytical Verification:}

Analytical verification of the relationship between $O S P I$ and the damping ratio is carried out here considering two cases: A single machine infinite bus (SMIB) system with second order synchronous machine model and a three bus test system with 
third order synchronous machine model, as described below. The relationship between the OSPI and damping ratio of the critical mode can be easily identified on a SMIB system with the generator represented by the second order model. The infinite bus is considered as the reference bus. Therefore, the OSPI simply becomes machine angle $\delta$. The damping ratio of the oscillatory mode associated with complex Eigenvalues can be approximated by (14) [25].

$$
\xi \approx \frac{1}{4 H} \frac{k_{D}}{\sqrt{\left\{\frac{\omega E \cos \delta}{2 X H}\right\}}}
$$

It is evident that the higher the $\delta$ the higher the damping ratio. It proves that a higher OSPI indicates a better oscillatory stable system. This relationship can further verify analytically with a SIMB and a load bust test system shown in Fig. 1, with the generator represented by the third order model, which incorporates field circuit dynamics.

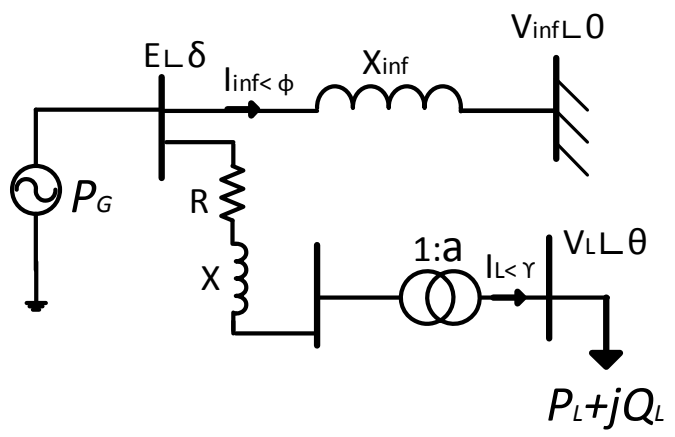

Fig. 1. Single machine infinite and load bus test system.

The linearized system state equations are derived following the methodology given in [5]. The linearized system state equations can be described in the following form, with the parameter defined in Appendix.

$\left[\begin{array}{l}\Delta \dot{\omega}_{r} \\ \Delta \dot{\delta} \\ \Delta \dot{\Psi}_{f d}\end{array}\right]=\left[\begin{array}{ccc}-\frac{K_{D}}{2 H} & -\frac{K_{T \delta}}{2 H} & -\frac{K_{T \Psi}}{2 H} \\ \omega_{0} & 0 & 0 \\ 0 & K_{\Psi \delta} & K_{\Psi \Psi}\end{array}\right]\left[\begin{array}{l}\Delta \omega_{r} \\ \Delta \delta \\ \Delta \Psi_{f d}\end{array}\right]+\left[\begin{array}{ll}\frac{1}{2 H} & 0 \\ 0 & 0 \\ 0 & K_{E f d}\end{array}\right]\left[\begin{array}{l}\Delta T_{m} \\ \Delta E_{f d}\end{array}\right]$

The generator data is given in Appendix- Table A-I. The system data incorporated in the analysis for $X_{i n f}, V_{i n f}, P_{G}, X_{t}, X, R_{L}, a$ and $E$ are 0.4 p.u., 1 p.u., 1 p.u., 0.15 p.u., 0.1 p.u., 0.15 p.u., 1 and 1.08 p.u., respectively. The load is having a fixed power factor of 0.95 lagging. Its magnitude is varied from $0.2-0.3$ p.u. to obtain different system operating points.

The damping ratio of the critical modes and the total of system bus angle deviations with respect to the reference bus angle $(O S P I)$ are calculated for different operating points. The results are shown in Fig. 2.

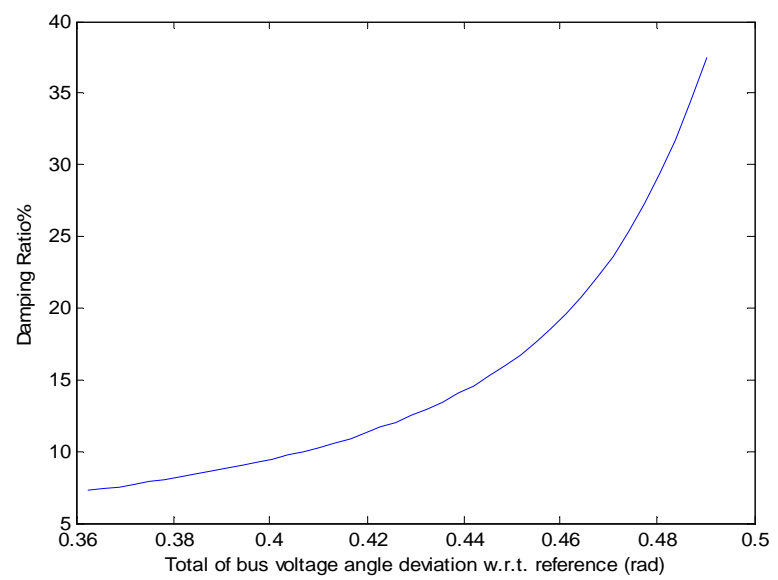

Fig. 2. The relationship between the total bus angle deviation (OSPI) and the damping ratio of the critical mode.

It is clear that the higher the total bus voltage angle deviations with respect to the reference bus voltage angle (OSPI) the higher the damping ratio. It proves $O S P I$ is a good indicator of system damping status. Further, numerical investigations are carried out in IEEE 14 bus test system.

\section{2) Numerical Verification:}

The Eigenvalue analysis and power flow analysis of the IEEE 14 bus test system are carried out using power system analysis tool (PSAT). The network data is given in [32]. The original network loads were reduced by $20 \%$. A 0.2 p.u. load, having 0.95 lagging power factor is connected at different network busses, during each simulation. The generators are modeled with sixth order dynamic models and with type two (IEEE model 1) exciters. The simulation results are shown in Table I. The load connected busses are ranked according to impact on oscillatory stability. The obtained damping ratio associated with the critical modes and the total of individual bus angle deviations with respect to infinite bus $(O S P I)$ are plotted in Fig. 3.

TABLE I. THE SIMULATION RESULTS

\begin{tabular}{|c|c|c|c|}
\hline Bus Number & $\begin{array}{c}\text { Damping ratio } \\
(\%)\end{array}$ & OSPI & $\begin{array}{c}\text { Bus ranking } \\
\text { (Best to worse) }\end{array}$ \\
\hline 6 & 4.505 & -2.649 & 1 \\
\hline 9 & 4.487 & -2.658 & 2 \\
\hline 10 & 4.445 & -2.678 & 3 \\
\hline 13 & 4.398 & -2.687 & 4 \\
\hline 14 & 4.308 & -2.709 & 5 \\
\hline
\end{tabular}

It is evident that there is a direct relationship between damping ratio of the critical mode and the total bus voltage angle deviations with respect to the slack bus voltage angle (OSPI). The bus ranking based on the $O S P I$ is the same as bus ranking based on damping ratio.

Both the analytical and the numerical studies indicate that there is a direct relationship between damping ratio of the critical mode and $O S P I$. 


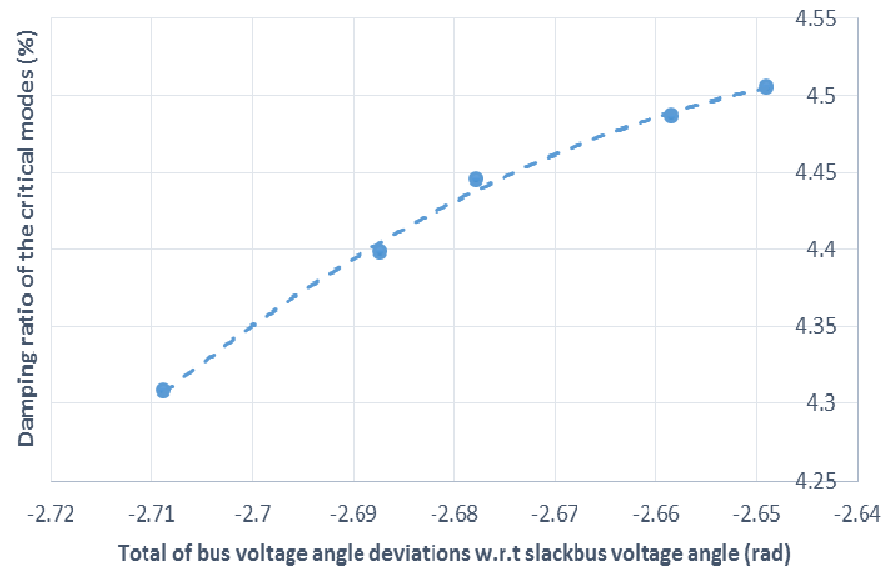

Fig. 3. The relationship between the total bus angle deviation and the damping ratio of the critical mode.

Further, it is evident that more positive total angle deviation is better. This indicates a lesser stress to the main grid as the load is partially met by the distributed resources, with reduced line flows and power losses. On the other hand, more negative total angle deviations with respect to the slack bus means higher stress on the main grid and its generators. That will cause higher impact on oscillatory stability. Therefore, OSPI can be maximized during planning study to identify better oscillatory stable planning solution. OSPI is calculated based on load flow results, hence involves less computational efforts. Although, the index cannot quantify the system stability status, identification of a better solution will minimize the risk of system become unstable due to a vulnerable operating point.

\section{B. Index for voltage stability}

Studies have identified that EV charging load can deteriorate power system voltage stability $[4,6,7]$. Therefore, incorporating system voltage stability within the EV charging infrastructure planning process is of great importance. Drawbacks of currently available techniques in utilizing planning studies have been thoroughly discussed in [33]. The importance of identifying simple and computationally efficient index yet having good physical interpretations has been identified. This paper numerically validates the suitability of previously derived index $Q R P I$ in [33], for identifying a relatively better voltage stable planning solution.

The evaluated index is based on the available reactive power reserve of the system. Reactive power resource management plays a big role in system planning and operation to maintain voltage stability of a power system. The reactive power reserve (RPR) preserves the ability of a power system to act upon contingencies. The reactive power reserve has been calculated with respect to the maximum reactive power limit of the reactive power sources. The derived $Q R P I$ index in [33] is described in (15).

$$
Q R P I=\sum_{i=1}^{N_{Q r}}\left\{W_{i} \frac{\left(Q_{R, i, \text { base }}-Q_{R, i}\right)}{Q_{R, i, \text { base }}}\right\} \quad \forall W_{i} \in(0,1), \quad \sum_{i=1}^{l} W_{i}=1
$$

The $Q_{R, i, b a s e}$ is the RPR of the generator $i$ at the base case, while $Q_{R, i}$ is the RPR of the generator $i$ in a particular planning case. Normalization is incorporated within the index to give more emphasis to the generators which are having smaller RPR (or which are about to reach the maximum reactive power limit) at the base case. A weight $\left(W_{i}\right)$ has been assigned to each reactive power resource to indicate its relative importance in maintaining system voltage stability. A higher weight is given to more critical resources so that those will utilize least during a planning exercise. Thus, placement of the EV load at locations where the $Q R P I$ is lower will lower the impact on system voltage stability.

\section{1) Numerical Verification of QRPI:}

A validation of the proposed $Q R P I$ model has been carried out in the IEEE 14 bus test system. The network data is given in [32]. The existing network loads are reduced by $20 \%$. The loading margin, reactive power reserve, the weighted reactive power reserve and the $Q R P I$ are evaluated when $2 \mathrm{MW}$ load having 0.95 lagging power factor is connected at different network busses.

Each reactive power resource is weighed based on the sequence they reach their reactive power limit during CPF carried out for the base case. Higher weights are assigned to the sources which reach the reactive power limit first, as shown in Table II. The simulation results are shown in Table III. The relationship between the loading margin and the $Q R P I$ is illustrated in Fig. 4.

TABLE II. The Weights Assigned to EACH ReACtive Power SOURCE

\begin{tabular}{|c|c|}
\hline Bus Name & Assigned weights \\
\hline 1 & 0.1 \\
\hline 2 & 0.15 \\
\hline 3 & 0.2 \\
\hline 6 & 0.25 \\
\hline 8 & 0.3 \\
\hline
\end{tabular}

TABLE III. THE SiMULATION RESUlTS

\begin{tabular}{|c|c|c|c|c|c|}
\hline $\begin{array}{c}\text { Bus } \\
\text { Name }\end{array}$ & $\begin{array}{c}\text { Loading } \\
\text { margin } \\
\text { (loading } \\
\text { parameter) }\end{array}$ & $\begin{array}{c}\text { Reactive } \\
\text { power } \\
\text { reserve } \\
\text { (Mvar) }\end{array}$ & $\begin{array}{c}\text { Weighted } \\
\text { reactive } \\
\text { power } \\
\text { reserve } \\
\text { (Mvar) }\end{array}$ & $\begin{array}{c}\text { QRPI } \\
\%\end{array}$ & $\begin{array}{c}\text { Bus } \\
\text { ranking } \\
\text { (Best to } \\
\text { worse) }\end{array}$ \\
\hline 6 & 2.12 & 10.55632 & 1.112974 & 0.3005 & 1 \\
\hline 9 & 2.1134 & 10.55529 & 1.112829 & 0.3135 & 2 \\
\hline 10 & 2.1105 & 10.55511 & 1.112769 & 0.3189 & 3 \\
\hline 14 & 2.0942 & 10.55422 & 1.112532 & 0.3401 & 4 \\
\hline
\end{tabular}

It is clear from the simulation results that there is a direct relationship between $Q R P I$ and loading margin. The bus ranking based on the $Q R P I$ is the same as bus ranking based on loading margin. Hence, $Q R P I$ based on load flow analysis can be incorporated to determine relative voltage stability between different planning solutions efficiently with less computational efforts. 


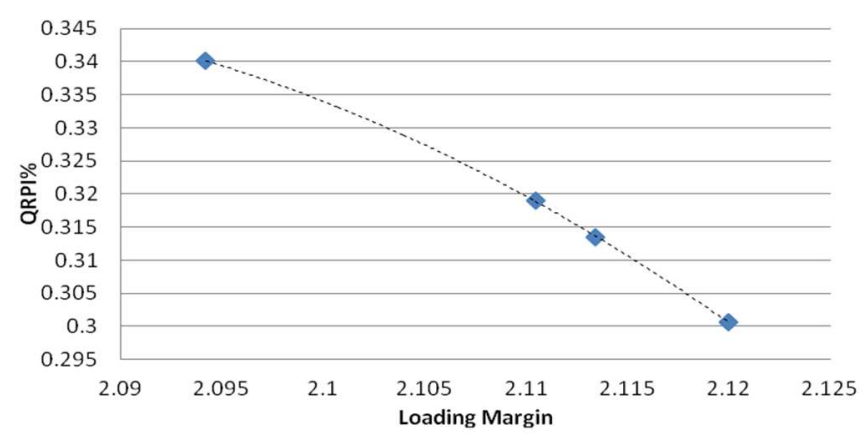

Fig. 4. The relationship between QRPI and loading margin.

\section{Planning EV CHARgING INFRASTRUCTURE}

\section{A. Problem formulation}

A number of planning strategies have been introduced in recent literatures for a successful transformation to an electrified transportation. It is evident that most of the planning objectives are traditionally aiming to satisfy the requirements of EV consumers or investors [11, 12, 15, 20-24]. The study in [34] describes how voltage stability can be incorporated within a planning study. This study focuses on planning charging infrastructure which not only place less impact on both system oscillatory stability and voltage stability, but also satisfies the investor and the customer requirements.

The particle swarm optimization tool is utilized for problem solving. As EV load being a voltage dependent load the Newton Rapson power flow algorithm should be slightly modified to accommodate load voltage dependency. The power flow equations and the diagonal elements of the power flow Jacobean matrix are modified as described in [33].

A multi-objective planning framework is proposed to identify a customer, investor and power grid concerned charging infrastructure. The optimization problem is formulated to maximize $O S P I$ and to minimize $Q R P I$, real and reactive power losses $\left(P_{\text {Loss }}\right.$ and $\left.Q_{\text {Loss }}\right)$ and charging station investment cost $\left(C_{\text {invest }}\right)$. Further, the total number of charger integrations $\left(C h_{t o t}\right)$ need to be maximized, while the charger allocations are effectively following the demand distribution in the area under consideration ( measured by $D_{p l}$ ). The index $D_{p l}$ measure the deviation of charger placement $\left(C h_{k}\right)$ from the desired placement $\left(\tau_{k} C h_{t o t}\right)$ considering the importance of the site $\left(\tau_{k}\right)$, as shown in (16). Hence, $D_{p l}$ needed to be minimized during planning.

$$
D_{p l}=\frac{1}{N_{s}} \sum_{k=1}^{N s}\left|\tau_{k} C h_{t o t}-C h_{k}\right| \quad \forall \tau_{k} \in(0,1), \quad \sum_{i=1}^{N s} \tau_{k}=1
$$

Each individual objective $\left(\mu_{i}\right)$ is normalized before fixing into the final objective function, following the methodology given in [34]. The weighed aggregated method described in (17) is incorporated to build the objective function shown in (18).

The objective function;

$$
\begin{aligned}
& F=\operatorname{Min} \sum_{i=1}^{i}\left(\bar{\mu}_{i} * \sigma_{i}\right) \quad \forall \sigma_{i} \in(0,1), \quad \sum_{i=1}^{i} \sigma_{i}=1 \\
& F=\operatorname{Min}\left\{\sigma_{1} \bar{P}_{\text {Loss }}+\sigma_{2} \bar{Q}_{\text {Loss }}+\sigma_{3} \bar{D}_{p l}+\sigma_{4} \overline{O S P I}+\sigma_{5} \bar{C}_{\text {invest }}+\sigma_{6} \bar{C} h_{\text {tot }}+\sigma_{7} \overline{Q R P I}\right\}
\end{aligned}
$$

Further, the following constraints are incorporated.

The problem constraints;

- The load bus voltages should be maintained within $\pm 6 \%$.

- The power lines are kept within $90 \%$ of the thermal rating.

- $Q_{R, i}$ is not allowed fall below the $90 \%$ of the base case RPR $\left(Q_{R, i, b a s e}\right)$.

- The number of allocated charging slots at each site should be kept within the site's minimum required and the site's maximum possible.

Two planning scenarios are introduced for comparison purpose. The Scenario 1 described the best placement in the grid point of view, while the Scenario 2 is the most comprehensive one, which consider requirements of customer, investor and grid operator, as described below.

Scenario 1-The main concern is the power grid and it is required to place the maximum number of chargers to avoid future unplanned charger integrations while causing the lowest impact to the power system in terms of system stability and real and reactive power losses.

Scenario 2- this is a comprehensive planning strategy which considers to meet the requirements of EV consumer, the charging facility investor and the grid operator, optimally.

\section{B. Case Study}

A test case is created to identify optimal charger allocations for four potential EV demand sites. The locations are marked as S1-S4 in the IEEE 30 bus test system as shown in Fig. 5. The IEEE 30 bus test system load flow data and line MVA flow limits were obtained from [35] and [36], respectively. The individual chargers are rated at $100 \mathrm{~kW}$ and 0.95 lagging power factor.

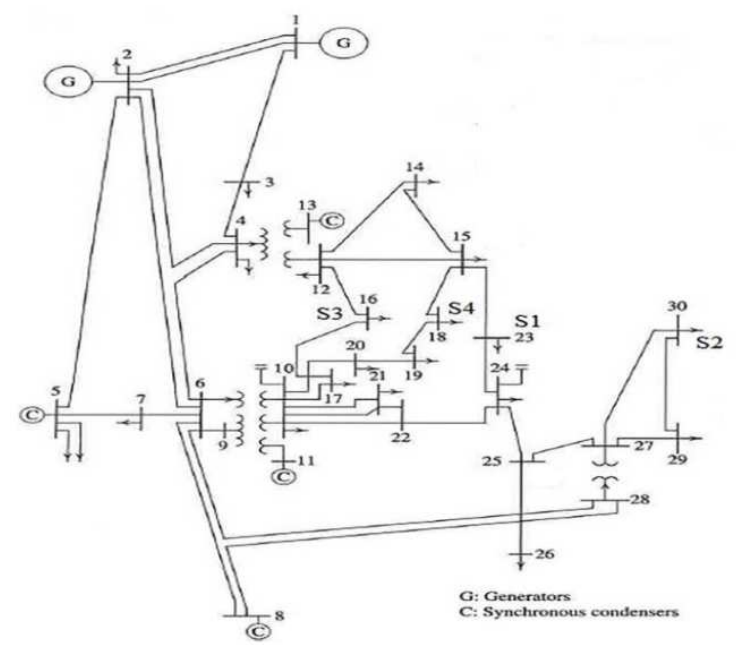

Fig. 5. The locations of the EV sites on IEEE 30 bus network diagram[35]. 
Each individual site is assigned with a weight $\left(\tau_{k}\right)$ to indicate its relative importance, as shown in Table IV. Further the assumed investment costs and the charger requirements in each individual site are also shown in TABLE IV.

TABLE IV. PLANNING DATA FOR THE EV SITES

\begin{tabular}{|l|c|c|c|c|}
\hline \multicolumn{1}{|c|}{ Site } & $\mathrm{S} 1$ & $\mathrm{~S} 2$ & $\mathrm{~S} 3$ & $\mathrm{~S} 4$ \\
\hline $\begin{array}{l}\text { Weight assigned to indicate } \\
\text { importance of the site }\left(\tau_{\mathrm{k}}\right)\end{array}$ & 0.3 & 0.35 & 0.2 & 0.15 \\
\hline Fixed investment cost & 1.265 & 0.195 & 0.557 & 1.094 \\
\hline $\begin{array}{l}\text { Variable investment cost/ } \\
\text { charging space }\end{array}$ & 1.812 & 1.629 & 1.527 & 1.354 \\
\hline Min required charging spaces & 1 & 1 & 1 & 1 \\
\hline $\begin{array}{l}\text { Max allowable charging } \\
\text { spaces }\end{array}$ & 30 & 30 & 30 & 30 \\
\hline
\end{tabular}

A random weight is assigned to each reactive power resource to indicate its relative importance for the purpose of calculating QRPI. The values are given in Table V.

TABLE V. Weights Assigned to Each Reactive Power Source

\begin{tabular}{|l|c|c|c|c|c|}
\hline \multicolumn{1}{|c|}{ Q resource } & G2 & C5 & C8 & C11 & C13 \\
\hline $\begin{array}{l}\text { Weight } \\
\text { assigned }\end{array}$ & 0.3 & 0.15 & 0.1 & 0.25 & 0.2 \\
\hline
\end{tabular}

Initially, the PSO program is executed to get the maximum and minimum of each individual objective, subjected to the problem constraints, the results are shown in Table VI. These maximum and minimum values will be utilized to normalize the individual objectives before fixing to the final minimization function.

TABLE VI. The MAXimums AND the Minimums of the OBJeCtives

\begin{tabular}{|l|c|c|c|c|c|c|l|}
\hline Objective & $\begin{array}{c}P_{\text {Loss }} \\
/(\mathrm{MW})\end{array}$ & $\begin{array}{c}\mathrm{Q}_{\text {Loss }} \\
/ \text { (MVar) }\end{array}$ & $\mathrm{D}_{\mathrm{pl}}$ & OSPI & QRPI & $\begin{array}{c}\mathrm{C}_{\text {invest }} \\
/(\text { Units })\end{array}$ & $\mathrm{Ch}_{\text {tot }}$ \\
\hline Minimum & 17.6807 & 22.5963 & 0 & -7.614 & 0.0521 & 11.062 & 5 \\
\hline Maximum & 18.1517 & 24.4436 & 10.325 & -7.491 & 0.3099 & 55.446 & 31 \\
\hline
\end{tabular}

The grid preferred scenario (scenarios 1), consider a placement which results lowest grid impacts. It also considers placing maximum chargers in their favored locations to avoid future abrupt charger placements. The grid stability is foremost important compared to grid real and reactive power loss, therefore relatively high weights are allocated to $Q R P I$ and $O S P I$ compared to $P_{\text {Loss }}$ and $Q_{\text {Loss }}$. The comprehensive planning scenario (scenarios 2 ) gives priority to accommodate maximum number of chargers according to demand distribution, followed by a more grid stable and a lesser expensive solution. The other objectives are weighted less as those are lesser significant in the final overall objective. The weights assigned to each objective, in two planning Scenarios are shown in Table VII. The planning outcomes are shown in Table VIII.

TABLE VII. The WeIGHTS GIVEN to INDIVIDUAL OBJectives

\begin{tabular}{|l|l|l|l|l|l|l|l|}
\hline Scenario & $\mathrm{P}_{\text {Loss }}$ & $\mathrm{Q}_{\text {Loss }}$ & $\mathrm{D}_{\mathrm{pl}}$ & OSPI & $\mathrm{QRPI}$ & $\mathrm{C}_{\text {invest }}$ & $\mathrm{Ch}_{\text {tot }}$ \\
\hline Scenario 1 & 0.10 & 0.08 & 0 & 0.18 & 0.18 & 0 & 0.46 \\
\hline Scenario 2 & 0.08 & 0.04 & 0.12 & 0.10 & 0.10 & 0.10 & 0.46 \\
\hline
\end{tabular}

It is evident from the results that scenario 1 has identified a grid preferred solution as indicated by lower real and reactive power losses and higher $O S P I$, even for a higher number of EV integrations. However, it is not an EV customer and the investor satisfied solution as it has not followed the EV demand distribution which is indicated by a higher $D_{p l}$. The scenario 2 provides a better solution with an overall objective satisfaction.

TABLE VIII. THE PLANNING RESUlts

\begin{tabular}{|l|l|l|}
\hline Objective values & Scenario 1 & Scenario 2 \\
\hline $\mathrm{P}_{\text {Loss }} /(\mathrm{MW})$ & 18.0166 & 18.0747 \\
\hline $\mathrm{Q}_{\text {Loss }}(\mathrm{MVar})$ & 24.2334 & 24.2958 \\
\hline $\mathrm{D}_{\mathrm{pl}}$ & 10.4 & 0.25 \\
\hline OSPI & -7.6018 & -7.6067 \\
\hline $\mathrm{QRPI}$ & 0.3094 & 0.3032 \\
\hline $\mathrm{C}_{\text {invest }} /$ (Units) & 50.7640 & 50.2870 \\
\hline $\mathrm{Ch}_{\text {tot }}$ & 31 & 29 \\
\hline Charger distribution S1-S4 & $1,2,27,1$ & $9,10,6,4$ \\
\hline
\end{tabular}

It satisfies the investor and the grid operator moderately. It has followed the demand distribution satisfactorily as described by a lower $D_{p l}$. Hence, it will provide a customer satisfied solution.

\section{CONCLUSIONS}

Evaluation of system stability status in planning studies is an important task. Two indexes have been evaluated which can be utilized to determine relative voltage and oscillatory stability status. The OSPI index, which based on the total of individual bus voltage angle deviations with respect to the reference bus angle is in good agreement with the damping ratio of the critical mode, while $Q R P I$ based on system reactive power reserve follows the system loading margin. Hence, the indexes $O S P I$ and $Q R P I$, which are based on load flow results, can efficiently incorporate in steady state system planning programs to determine the relative voltage and oscillatory stability status in different planning options.

A multi-objective planning framework has been developed here to achieve satisfied planning solution for customer, investor and grid operator. The OSPI and QRPI indexes could easily integrate into planning algorithms to identify better planning solutions. Hence, these indexes can be incorporated in panning studies which are based on steady state system analysis, to identify stability cogitated planning solutions.

\section{APPENDIX}

$K_{q \delta}=\frac{1}{n}\left\{E \cos \delta+\frac{E \sin \delta\left(L_{i}+L^{\prime}\right)}{R_{a}}\right\}$

$K_{q \psi}=-\frac{L^{\prime}}{n L_{f d}}$

$K_{d \delta}=\frac{1}{n}\left\{\frac{E \cos \delta\left(L_{i}+L_{a q}\right)}{R_{a}}-E \sin \delta\right\}$

$K_{d \psi}=-\frac{\left(L_{l}+L_{a q}\right) L^{\prime}}{n R_{a} L_{f d}}$

$K_{\psi q d}=-L_{a q} K_{q \delta}$

$K_{\psi q \delta}=-L_{a q} K_{q \delta}$ 


$$
\begin{aligned}
& K_{\psi q \psi}=-L_{a q} K_{q \psi} \\
& K_{\psi d \delta}=-L^{\prime} K_{d \delta} \\
& K_{\psi d \psi}=\frac{L^{\prime}}{L_{f d}}-K_{d \psi} \\
& K_{T \delta}=\psi_{a d 0} K_{q \delta}+i_{q 0} K_{\psi d \delta}-\psi_{a q 0} K_{d \delta}-i_{d 0} K_{\psi q \delta} \\
& K_{T \psi}=\psi_{a d 0} K_{q \psi}+i_{q 0} K_{\psi d \psi}-\psi_{a q 0} K_{d \psi}-i_{d 0} K_{\psi q \psi} \\
& K_{\psi \delta}=\frac{\omega_{0} R_{f d} K_{\psi d \delta}}{L_{f d}} \\
& K_{\psi \psi}=\frac{\omega_{0} R_{f d} K_{\psi d \psi}}{L_{f d}}-\frac{\omega_{0} R_{f d}}{L_{f d}}
\end{aligned}
$$

TABLE A-I. SYNCHRONOUS MACHINE DYNAMIC ORDER-3 DATA [25]

\begin{tabular}{|l|l|l|l|}
\hline Parameter & Value & Parameter & Value \\
\hline $\mathrm{Pg}$ & 1.0 p.u. & $\mathrm{X}$ & 0.40 p.u. \\
\hline $\mathrm{R}_{\mathrm{a}}$ & 0.003 p.u. & $\mathrm{R}_{\mathrm{fd}}$ & 0.0006 p.u. \\
\hline $\mathrm{L}_{\mathrm{l}}$ & 0.16 p.u. & $\mathrm{L}_{\mathrm{adu}}$ & 1.65 p.u. \\
\hline $\mathrm{L}_{\mathrm{aqu}}$ & 1.6 p.u. & $\mathrm{L}_{\mathrm{fd}}$ & 0.153 p.u. \\
\hline $\mathrm{H}$ & $3.5 \mathrm{~s}$ & $\mathrm{~K}_{\mathrm{d}}$ & 0 \\
\hline $\mathrm{K}_{\mathrm{sdincr}}$ & 0.434 & $\mathrm{~K}_{\mathrm{sqincr},}$ & 0.434 \\
\hline $\mathrm{K}_{\mathrm{sq}}$ & 0.8491 & $\mathrm{~K}_{\mathrm{sq}}$ & 0.8491 \\
\hline
\end{tabular}

\section{REFERENCES}

[1] I. IEA, "GLOBAL EV OUTLOOK," International Energy Agency 2013.

[2] C. Allcock, J. Barghout, and K. Drye, "EV City Case Book," 2012.

[3] C. H. Dharmakeerthi, N. Mithulananthan, and T. K. Saha, "Overview of the impacts of plug-in electric vehicles on the power grid," in Innovative Smart Grid Technologies Asia (ISGT), 2011 IEEE PES, 2011, pp. 1-8.

[4] C.H.Dharmakeerthi and N.Mithulananthan, "A Study of Load and Line Characteristics on Power System Damping Performance " presented at the IEEE 8th International Conference on Industrial and Information Systems, Kandy, Sri Lanka, 2013.

[5] C.H.Dharmakeerthi, N.Mithulananthan , and T.K.Saha, "Impact of Electric Vehicle Load on Power System Oscillatory Stability," presented at the The Australasian Universities Power Engineering Conference, Tasmania, 2013.

[6] C. H. Dharmakeerthi, N. Mithulananthan, and T. K. Saha, "Modeling and planning of EV fast charging station in power grid," in Power and Energy Society General Meeting, 2012 IEEE, 2012, pp. 1-8.

[7] C. H. Dharmakeerthi, N. Mithulananthan, and T. K. Saha, "Impact of electric vehicle fast charging on power system voltage stability," International Journal of Electrical Power \& Energy Systems, vol. 57, pp. 241-249, 2014.

[8] T. Das and D. C. Aliprantis, "Small-Signal Stability Analysis of Power System Integrated with PHEVs," in Energy 2030 Conference, 2008. ENERGY 2008. IEEE, 2008, pp. 1-4.

[9] H. Huang, C. Y. Chung, K. W. Chan, and H. Chen, "Quasi-Monte Carlo Based Probabilistic Small Signal Stability Analysis for Power Systems With Plug-In Electric Vehicle and Wind Power Integration," Power Systems, IEEE Trans. on, vol. PP, pp. 1-9, 2013.

[10] F. R. Islam, H. R. Pota, M. A. Mahmud, and M. J. Hossain, "Impact of PHEV loads on the dynamic performance of power system," in Universities Power Engineering Conference (AUPEC), 2010 20th Australasian, 2010, pp. 1-5.

[11] J. Long, H. Zechun, S. Yonghua, and L. Zhuowei, "Optimal siting and sizing of electric vehicle charging stations," in Electric Vehicle Conference (IEVC), 2012 IEEE International, 2012, pp. 1-6.

[12] Dharmakeerthi C.H., Mithulananthan N., and S. T.K., "Planning of Electric Vehicle Charging Infrastructure " presented at the Power and Energy Society General Meeting, 2013 IEEE PES, 2013.

[13] Z. Lili, X. Ming, D. Jin, Z. Zhiyu, and W. Xilin, "Electric vehicle charging facility planning in Shenzhen Power Supply Bureau Limited
Company," in Electric Vehicle Conference (IEVC), 2012 IEEE International, 2012, pp. 1-5.

[14] J. Liu, "Electric vehicle charging infrastructure assignment and power grid impacts assessment in Beijing," Energy Policy, vol. 51, pp. 544-557, 2012.

[15] Z. Liu, F. Wen, and G. Ledwich, "Optimal Planning of Electric-Vehicle Charging Stations in Distribution Systems," Power Delivery, IEEE Trans on, vol. PP, pp. 1-1, 2012.

[16] A. H. Hajimiragha, C. A. Canizares, M. W. Fowler, S. Moazeni, and A. Elkamel, "A Robust Optimization Approach for Planning the Transition to Plug-in Hybrid Electric Vehicles," Power Systems, IEEE Trans. on, vol. 26, pp. 2264-2274, 2011.

[17] L. Zhipeng, W. Fushuan, and G. Ledwich, "Optimal Planning of Electric-Vehicle Charging Stations in Distribution Systems," Power Delivery, IEEE Trans. on, vol. 28, pp. 102-110, 2013.

[18] Y. Li, L. Li, J. Yong, Y. Yao, and Z. Li, "Layout Planning of Electrical Vehicle Charging Stations Based on Genetic Algorithm," in Electrical Power Systems and Computers. vol. 99, X. Wan, Ed., ed: Springer Berlin Heidelberg, 2011, pp. 661-668.

[19] G. Wang, Z. Xu, F. Wen, and K. P. Wong, "Traffic-Constrained Multiobjective Planning of Electric-Vehicle Charging Stations," Power Delivery, IEEE Transactions on, vol. 28, pp. 2363-2372, 2013.

[20] T. Xiangang, L. Junyong, W. Xiaoyin, and X. Jie, "Electric vehicle charging station planning based on weighted Voronoi diagram," in Transportation, Mechanical, and Electrical Engineering (TMEE), 2011 International Conference on, 2011, pp. 1297-1300.

[21] Z.-f. Liu, W. Zhang, X. Ji, and K. Li, "Optimal Planning of charging station for electric vehicle based on particle swarm optimization," in Innovative Smart Grid Technologies - Asia (ISGT Asia), 2012 IEEE, 2012, pp. 1-5.

[22] F. Liang, G. Shaoyun, L. Hong, W. Long, and F. Yan, "The planning of charging stations on the urban trunk road," in Innovative Smart Grid Technologies - Asia (ISGT Asia), 2012 IEEE, 2012, pp. 1-4.

[23] G. Shaoyun, F. Liang, and L. Hong, "The planning of electric vehicle charging station based on Grid partition method," in Electrical and Control Engineering (ICECE), 2011 International Conference on, 2011, pp. 2726-2730.

[24] F. Liang, G. Shaoyun, and L. Hong, "Electric Vehicle Charging Station Planning Based on Weighted Voronoi Diagram," in Power and Energy Engineering Conference (APPEEC), 2012 Asia-Pacific, 2012, pp. 1-5.

[25] P. Kundur, Power System Stability and Control: McGraw-Hill, 1994.

[26] K. Prasertwong, N. Mithulananthan, and D. Thakur, "Understanding low frequency oscillation in power systems," International Journal of Electrical Engineering Education vol. 47, p. 15, 2010.

[27] IEEE/PES, "Voltage Stability Assessment Procedures and Guides," Power System Stability Subcommittee2001

[28] K. E. Reinhard, P. W. Sauer, and A. D. Dominguez-Garcia, "On Computing Power System Steady-State Stability Using Synchrophasor Data," in System Sciences (HICSS), 2013 46th Hawaii International Conference on, 2013, pp. 2312-2318.

[29] R. F. Nuqui, A. G. Phadke, R. P. Schulz, and N. Bhatt, "Fast on-line voltage security monitoring using synchronized phasor measurements and decision trees," in Power Engineering Society Winter Meeting, 2001. IEEE, 2001, pp. 1347-1352 vol.3.

[30] L. Guang and S. M. Rovnyak, "Integral square generator angle index for stability ranking and control," Power Systems, IEEE Trans. on, vol. 20, pp. 926-934, 2005 .

[31] S. M. Rovnyak, M. N. Nilchi, D. W. Longbottom, and D. C. Vasquez, "Angle stability predictive indices," in Power and Energy Society General Meeting, 2012 IEEE, 2012, pp. 1-6.

[32] F. Milano, "Power System Analysis Toolbox Documentation for PSAT version 1.3.4, July 14, 2005," 2005

[33] C.H.Dharmakeerthi, N. Mithulananthan, and T. K. Saha, "A Comprehensive Planning Framework for Electric Vehicle Charging Infrastructure Deployment in the Power Grid with Enhanced Voltage Stability," International Transactions on Electrical Energy Systems, 2014.

[34] C.H.Dharmakeerthi, N.Mithulananthan, and T.K.Saha, "Planning of Electric Vehicle Charging Infrastructure " presented at the PESGM, Vancouver, British Columbia, Canada., 2013.

[35] H. Saadat, Power System Analysis, 3 ed.: PSA Publishing, 2010.

[36] M. Shahidehpour and Y. Wang, "Appendix C: IEEE-30 Bus System Data," in Communication and Control in Electric Power Systems, ed: John Wiley \& Sons, Inc., 2005, pp. 493-495. 
Dr. Mithulan received his B.Sc. (Eng.) and M. Eng. degrees from the University of Peradeniya, Sri Lanka, and the Asian Institute of Technology (AIT), Bangkok, Thailand, in May 1993 and August 1997, respetively. His Ph.D. degree from University of Waterloo, Ontario, Canada. Prior to joining the University of Queensland from 2009, Mithulan was attached to Energy Field of Study at AIT as an Associate Professor. His previous professional positions include: Planning Engineer at Generation Planning division for 2 years at Ceylon Electricity Board (CEB), Sri Lanka and Project Leader, at the Centre of Excellence in Electric Power Technology (CEEPT) for one year at Chulalongkorn University, Thailand. His main areas of research interests are analytical studies on electric power grids, power system interconnections, application of FACTS devices in power system, integration of renewable energy and Electric Vehicle. Based on his research, he published over 200 articles, including one book, book chapters, technical reports, refereed journal and conference papers
He was invited to join the editorial board of American Institute of Mathematical Sciences Electronic Engineering Journal-an international open access journal. $\mathrm{He}$ is a senior member of IEEE

C.H Dharmakeerthi received her B.Eng.Sc degree with first class honor and M.Sc. degree from the University of Peradeniya, Sri Lanka in 2005 and 2008, resepectively. Her Ph.D degree in Electrical Engineering earned from the University of Queensland, Brisbane, Australia, in 2014. Currently, Dr. Champa is Chief Electrical Engineer the Ceylon Electric Board Trainning Center, Castlereagh, Sri Lanka. She is also a member of the Institution of Engineering and Technology (UK) (MIET), Associate Member of the Institution of Engineers, Sri Lanka (AMIESL), and also Chartered Engineer, Engineering Council, UK (CEng). Dr. Champa research interest are Electrical Vehicle Grid Integration and Renewable Energy. 